\title{
Chemostat Model of Competition between Plasmid-Bearing and Plasmid-Free Organism with the Impulsive State Feedback Control
}

\author{
Fengmei Tao, ${ }^{1}$ Zhong Zhao $\mathbb{D D}^{2}{ }^{2}$ and Lansun $\mathrm{Chen}^{3}$ \\ ${ }^{1}$ School of Mathematics and Information Science, Anshan Normal University, Anshan 114007, Liaoning, China \\ ${ }^{2}$ Department of Mathematics, Huanghuai University, Zhumadian 463000, Henan, China \\ ${ }^{3}$ Academy of Mathematics and Systems Science, Chinese Academy of Sciences, Beijing 100080, China \\ Correspondence should be addressed to Zhong Zhao; zhaozhong8899@163.com
}

Received 27 June 2018; Accepted 16 September 2018; Published 23 October 2018

Academic Editor: Josef Diblík

Copyright (c) 2018 Fengmei Tao et al. This is an open access article distributed under the Creative Commons Attribution License, which permits unrestricted use, distribution, and reproduction in any medium, provided the original work is properly cited.

\begin{abstract}
In this paper, we propose a chemostat model of competition between plasmid-bearing and plasmid-free organism with the impulsive state feedback control. The sufficient condition for existence of the positive period-1 solution is obtained by means of successor function and the qualitative properties of the corresponding continuous system. We show that the impulsive control system is more effective than the corresponding continuous system if we choose a suitable threshold value of the state feedback control in the process of manufacturing the desired products through genetically modified techniques. Furthermore, a new method of proving the stability of the order-1 periodic solution is given based on the theory of the limit cycle of the continuous dynamical system. Finally, mathematical results are justified by some numerical simulations.
\end{abstract}

\section{Introduction}

With the rapid development of biotechnology, manufacturing the desired products through genetically modified techniques has been widely applied in many fields, such as agriculture, industrial biotechnology, and medicine. In general, genetical alteration is carried out by the introduction of a recombinant DNA into the cell in the form of a plasmid [1]. However, the plasmid may be lost in the reproductive process, which will introduce the plasmid-free organism into the process [2]. The genetically altered organism (plasmid-bearing organism) is less competitor than the plasmid-free organism because of the burden imposed by production [3]. At the same time, plasmid-free organism not only consumes the nutrient but also reduces the efficiency of the desired products. Therefore, the key problem is how to improve the efficiency of the desired products and to reduce the negative effect of the plasmid-free organism on the plasmid-bearing organism. Researchers [2-8] have done a lot of work to reduce the negative effect of the plasmid-free organism on the production efficiency of the desired products. In [2], a selective media was added from an external source and authors investigated the global stability of the equilibria by using the Dulac criterion and the Poincaré-Bendixson Theorem. Ai [5] introduced an inhibitor into the model of competition between plasmidbearing and plasmid-free organisms to reduce the effect of the plasmid-free organism on the plasmid-bearing organism. Yuan et al. [6] proposed a model for competition between plasmid-bearing and plasmid-free organisms in the chemostat with an external inhibitor and obtained some sufficient conditions of global attractivity to the extinction equilibria by constructing appropriate Lyapunov-like functionals. Based on a feedback control, Dimitrova [9] formulated a dynamical model of plasmid-bearing, plasmid-free competition in the chemostat with general specific growth rates, and global stabilization of the dynamics towards a practically important coexistence equilibrium point is obtained. Shi et al. [10] investigated a chemostat model with plasmid-bearing, plasmidfree competition and impulsive effect, and they obtained the invasion threshold of the plasmid-free organism and plasmid-bearing organism. 
Recently, impulsive state feedback control has been widely used in chemostat model [11-14]. Tian et al. [12] studied the dynamics of the bioprocess with the impulsive state feedback control and obtained the existence and stability of period-1 solution of the bioprocess. In [13], authors proposed a turbidostat model with the feedback control, and the sufficient conditions of existence of positive order-1 periodic solution were obtained by using the existence criteria of periodic solution of a general planar impulsive autonomous system. As far as the authors know, little information is given about the introduction of the impulsive state feedback control into chemostat model of competition between plasmid-bearing and plasmid-free organism. In this paper, we will formulate a mathematical model of competition between plasmid-bearing and plasmid-free organism with the impulsive state feedback control so as to further improve the efficiency of the desired products in the process of the genetic alteration.

The paper is organized as follows: a mathematical model of competition between plasmid-bearing and plasmid-free organism with the impulsive state feedback control is proposed in Section 2. In Section 3, the qualitative analysis of system without impulsive control is given. Furthermore, the existence and stability of order-1 periodic solution are investigated in Section 4. Finally, we give some numerical simulations and a brief discussion.

\section{Model Description and Preliminaries}

In the process of manufacturing the desired products through genetically modified techniques, an important way to control the negative effect is to reduce the competitive advantage of the plasmid-free organism.

The process of the genetic alteration is complex because it includes a series of biological and chemical reactions. Therefore, understanding the dynamic mechanism of genetic alteration is the most important factor for improving the efficiency of the desired products. Luo et al. [15] formulated a model of plasmid-bearing and plasmid-free competition in a chemostat as follows:

$$
\begin{aligned}
\frac{d S}{d t} & =D\left(S^{0}-S\right)-\sigma_{1}(S) x_{1}-\sigma_{2}(S) x_{2}, \\
\frac{d x_{1}}{d t} & =x_{1}\left(f_{1}(S)(1-q)-D\right), \\
\frac{d x_{2}}{d t} & =x_{2}\left(f_{2}(S)-D\right)+q x_{1} f_{1}(S),
\end{aligned}
$$

where $S(t)$ is the nutrient concentration at time $t . x_{1}$ denotes the concentration of plasmid-bearing organisms at time $t$, and $x_{2}$ is the concentration of plasmid-free organisms at time $t$. The consumption rates and the specific growth rates of plasmid-bearing and plasmid-free organisms are $\sigma_{i}$ and $f_{i}$ $(\mathrm{i}=1,2)$, respectively. The probability that a plasmid is lost in reproduction is represented by $q(0<q<1)$.

Although the reason why plasmid is lost in the reproductive process is not completely understood by theoretical biologists even at present time, it has been experimentally validated that the plasmid-bearing cells have at least for some substrate concentrations a lower maximum specific growth rate than the plasmid-free counterpart $[9,16,17]$. To enhance the efficiency of the desired products and prevent the negative effect of the plasmid-free organism on the plasmidbearing organism, we try to find the critical threshold value (where plasmid-bearing cells reach the maximum specific growth rate), which can be obtained by the experiment. When the growth rate of the plasmid-free organism reaches the critical threshold value, we begin to extract the plasmid-free organism so as to control the concentration of the plasmidfree organism to be lower than the critical threshold value. Based on [11-14], we introduce the impulsive state feedback control into system (1):

$$
\begin{aligned}
\frac{d S}{d t} & =D\left(S^{0}-S\right)-\frac{\mu_{1} S x_{1}}{\delta}-\frac{\mu_{2} S x_{2}}{\delta}, \\
\frac{d x_{1}}{d t} & \left.=x_{1}\left(\mu_{1} S(1-q)-D\right)\right), \\
\frac{d x_{2}}{d t} & =x_{2}\left(\mu_{2} S-D\right)+\mu_{1} q S x_{1},
\end{aligned}
$$

$$
x_{2}<h
$$

$\Delta x_{1}=-E_{1} x_{1}$,

$\Delta x_{2}=-E_{2} x_{2}$,

$$
x_{2}=h,
$$

where $\mu_{1}$ and $\mu_{2}$ denote the maximal growth rate of the plasmid-bearing and plasmid-free organisms. $\delta$ is called a yield constant. $h$ is a critical threshold value which can be obtained by experiment. $E_{1}$ and $E_{2}\left(0<E_{i}<1, i=1,2\right)$ are the ratios of the plasmid-bearing and plasmid-free organisms extracted from the chemostat.

We firstly consider the qualitative property of (2) without the impulsive effect. The variables in the above system may be rescaled by measuring $S=S^{0} \widetilde{S}, x_{1}=\delta S^{0} x, x_{2}=\delta S^{0} y, t=$ $\tau / D$, and then system (2) becomes

$$
\begin{aligned}
& \frac{d \widetilde{S}}{d \tau}=1-\widetilde{S}-\frac{\mu_{1} S^{0} \widetilde{S} x}{D}-\frac{\mu_{2} S^{0} \widetilde{S} y}{D}, \\
& \frac{d x}{d \tau}=x\left(\frac{\mu_{1}(1-q) S^{0}}{D} \widetilde{S}-1\right), \\
& \frac{d y}{d \tau}=y\left(\frac{\mu_{2} S^{0} \widetilde{S}}{D}-1\right)+\frac{\mu_{1} q^{0} \widetilde{S} x}{D},
\end{aligned}
$$

$$
y<H,
$$

$$
\begin{aligned}
& \Delta x=-E_{1} x, \\
& \Delta y=-E_{2} y,
\end{aligned}
$$

$$
y=H,
$$

where $H=h / \delta S^{0}$.

Lemma 1. Suppose $\omega(\tau)=(\widetilde{S}(\tau), x(\tau), y(\tau))$ is a solution of (3) subject to $\omega\left(0^{+}\right) \geq 0$, and then $\omega(\tau) \geq 0$ for all $\tau \geq 0$, and further $\omega(\tau)>0, \tau \geq 0$ if $\omega\left(0^{+}\right)>0$. 
The proof is obvious. Hence we omit it.

From the first three equations, we have

$$
\frac{d \widetilde{S}}{d \tau}+\frac{d x}{d \tau}+\frac{d y}{d \tau}=1-\widetilde{S}-x-y
$$

which shows $\widetilde{S}+x+y=(\widetilde{S}(0)+x(0)+y(0)) e^{-\tau}+\left(1-e^{-\tau}\right)$ and we obtain

$$
\lim _{t \rightarrow \infty}(\widetilde{S}+x+y)=1
$$

From system (3) and (5), we obtain that the dynamical behavior of system (2) can be determined by the following system:

$$
\begin{aligned}
& \frac{d x}{d \tau}=x(A(1-q)(1-x-y)-1) \\
& \frac{d y}{d \tau}=y(B(1-x-y)-1)+A q(1-x-y) x,
\end{aligned}
$$$$
y<H
$$

$$
\begin{aligned}
& \Delta x=-E_{1} x, \\
& \Delta y=-E_{2} y,
\end{aligned}
$$

$$
y=H
$$

where $A=\mu_{1} S^{0} / D, B=\mu_{2} S^{0} / D$.

Definition 2 (see [18]). Let $M$ denote the impulsive set and $N$ be the phase set. Suppose that $g: N \longrightarrow N$ is a mapping. For any point $P \in N$, there exists a $t_{1}>0$ such that $F(\mathrm{P})=$ $f\left(P, t_{1}\right)=P_{1} \in M, P_{1}^{+}=\varphi\left(P_{1}\right) \in N$; then $g(P)=l\left(P_{1}^{+}\right)-l(P)$ is called the successor function of point $P$ and the point $P_{1}^{+}$is called the successor point of $P$.

\section{Qualitative Analysis}

We firstly consider the qualitative property of (6) without the impulsive effect.

$$
\begin{aligned}
\frac{d x}{d \tau} & =x(A(1-q)(1-x-y)-1)=P(x, y) \\
\frac{d y}{d \tau} & =y(B(1-x-y)-1)+A q(1-x-y) x \\
& =Q(x, y) .
\end{aligned}
$$

System (7) has three equilibria: $E_{0}(0,0), E_{1}(0,(B-1) / B)$, and a positive equilibrium $E_{2}\left(x^{*}, y^{*}\right)$,

$$
\begin{aligned}
& x^{*}=\frac{(A(1-q)-1)(A(1-q)-B)}{A(1-q)(A-B)}, \\
& y^{*}=\frac{A q x^{*}}{A(1-q)-B},
\end{aligned}
$$

where $A(1-q)>\max \{B, 1\}$.

Lemma 3. System (7) has no periodic trajectories in the positive quadrant.

Proof. We construct the Dulac function $F(x, y)=1 / x y$, and then we have

$$
\begin{aligned}
& \frac{\partial(P F)(x, y)}{\partial x}+\frac{\partial(F Q)(x, y)}{\partial y} \\
& =\frac{A(1-q)}{y}-\frac{B}{x}-\frac{A q(1-x)}{y^{2}}<0 .
\end{aligned}
$$

According to the Bendixson-Dulac Theorem [19], there is no periodic orbit in the positive quadrant.

Next, we discuss the stability of the equilibria. The Jacobian matrix evaluated at the point $E_{0}(0,0)$ is

$$
\left(\begin{array}{cc}
A(1-q) & 0 \\
A q & B-1
\end{array}\right)
$$

and the eigenvalues are $\lambda_{1}=A(1-q), \lambda_{2}=B-1$. Hence, the equilibrium $E_{0}(0,0)$ is saddle for $B>1$.

Theorem 4. The equilibrium $E_{1}(0,(B-1) / B)$ is globally asymptotically stable if $B>1$.

Proof. We can compute Jacobian matrix of the equilibrium $E_{1}(0,(B-1) / B)$ as follows:

$$
\left(\begin{array}{cc}
\frac{(1-q)}{B}-1 & 0 \\
1-B+\frac{A q}{B} & 1-B
\end{array}\right)
$$

and the eigenvalues are $\lambda_{3}=(1-q) / B-1, \lambda_{4}=1-B$. Obviously, the equilibrium $E_{1}(0,(B-1) / B)$ is asymptotically stable if $B>1$. Again from Lemma 3, the equilibrium $E_{1}(0,(B-1) / B)$ is globally asymptotically stable for $B>1$.

Theorem 5. The positive equilibrium $E_{2}\left(x^{*}, y^{*}\right)$ is globally asymptotically stable if $A(1-q)>\max \{B, 1\}$.

Proof. Firstly, we show the positive equilibrium $E_{2}\left(x^{*}, y^{*}\right)$ is locally asymptotically stable. Jacobian matrix of the equilibrium $E_{2}\left(x^{*}, y^{*}\right)$ is given as follows:

$$
J\left(E_{2}\left(x^{*}, y^{*}\right)\right)=\left(\begin{array}{cc}
-A(1-q) x^{*} & -A(1-q) x^{*} \\
-B y^{*}+A q\left(1-x^{*}-y^{*}\right)-A q x^{*} & B\left(1-x^{*}-y^{*}\right)-B y^{*}-A q x^{*}
\end{array}\right) .
$$


We perform a basic elementary column transformation for the matrix $J\left(E_{2}\left(x^{*}, y^{*}\right)\right)$ and obtain

$$
J^{\prime}\left(E_{2}\left(x^{*}, y^{*}\right)\right)=\left(\begin{array}{cc}
-A(1-q) x^{*} & 0 \\
-B y^{*}+A q\left(1-x^{*}-y^{*}\right)-A q x^{*} & (B-A q)\left(1-x^{*}-y^{*}\right)-1
\end{array}\right) .
$$

Hence, the eigenvalues of matrix $J\left(E_{2}\left(x^{*}, y^{*}\right)\right)$ have the same eigenvalues as the matrix $J^{\prime}\left(E_{2}\left(x^{*}, y^{*}\right)\right)$, and the eigenvalues are $\lambda_{5}=-A(1-q) x^{*}<0, \lambda_{6}=(B-A q)\left(1-x^{*}-y^{*}\right)-1=$ $(B-A) / A(1-q)<0$. In view of Lemma 3 , the equilibrium $E_{2}\left(x^{*}, y^{*}\right)$ is globally asymptotically stable if

$$
A(1-q)>\max \{B, 1\} .
$$

\section{Existence and Stability of the Order-1 Periodic Solution}

Case 1 (existence and stability of semitrivial periodic solution). Suppose $x=0$, and we have the following subsystem:

$$
\begin{aligned}
& \frac{d y}{d \tau}=y(B-1-B y), \quad y<H, \\
& \Delta y=-E_{2} y, \quad y=H .
\end{aligned}
$$

System (15) possesses a periodic solution $\eta(t)$ defined on $t \epsilon$ $(n \tau,(n+1) \tau]$ for $y\left(0^{+}\right)=\left(1-E_{2}\right) H$, where

$$
\begin{aligned}
& \eta_{2}(t) \\
& =\frac{(B-1) H\left(1-E_{2}\right) e^{(B-1)(t-n T)}}{(B-1)-B\left(1-E_{2}\right) H+B\left(1-E_{2}\right) H e^{(B-1)(t-n \tau)}} .
\end{aligned}
$$

If we let

$$
\begin{aligned}
T & =\frac{1}{B-1} \ln \frac{B-1-B\left(1-E_{2}\right) H}{(B-1-B H)\left(1-E_{2}\right)}, \\
y(T) & =h
\end{aligned}
$$

and $y\left(T^{+}\right)=\left(1-E_{2}\right) h$, which shows that system (6) has the following semitrivial periodic solution $(\xi(t), \eta(t))$, where

$$
\begin{aligned}
& \xi(t)=0, \\
& \eta(t) \\
& \quad=\frac{(B-1) H\left(1-E_{2}\right) e^{(B-1)(t-n \tau)}}{(B-1)-B\left(1-E_{2}\right) H+B\left(1-E_{2}\right) H e^{(B-1)(t-n \tau)}}
\end{aligned}
$$

for $t \in(n \tau,(n+1) \tau]$. Therefore, we obtain that $(0, \eta(t))$ is the semitrivial periodic solution of system (6).

Next we give one lemma firstly to discuss the stability of this semitrivial periodic solution of system (6).

Lemma 6. The $\tau$-periodic solution $x(t)=\xi(t), y(t)=\eta(t)$ of the system

$$
\begin{aligned}
& \frac{d x}{d t}=P(x, y), \\
& \frac{d y}{d t}=Q(x, y),
\end{aligned}
$$

$$
\phi(x, y) \neq 0,
$$

$$
\begin{aligned}
& \Delta x=\alpha(x, y), \\
& \Delta y=\beta(x, y),
\end{aligned}
$$

$$
\phi(x, y)=0
$$

is orbitally asymptotically stable if the Floquet multiplier $\mu_{2}$ satisfies the condition $\left|\mu_{2}\right|<1$, where

$$
\begin{aligned}
\mu_{2} & =\Pi_{k=1}^{q} \Delta_{k} \\
& \cdot \exp \left[\int_{0}^{\tau}\left(\frac{\partial P}{\partial x}(\xi(t), \eta(t))+\frac{\partial Q}{\partial y}(\xi(t), \eta(t))\right) d t\right],
\end{aligned}
$$

with

$$
\Delta_{k}=\frac{P_{+}((\partial \beta / \partial y)(\partial \phi / \partial x)-(\partial \beta / \partial x)(\partial \phi / \partial y)+(\partial \phi / \partial x))+Q_{+}((\partial \alpha / \partial x)(\partial \phi / \partial y)-(\partial \alpha / \partial y)(\partial \phi / \partial x)+(\partial \phi / \partial y))}{P(\partial \phi / \partial x)+Q(\partial \phi / \partial y)}
$$

and $P, Q, \partial \alpha / \partial x, \partial \alpha / \partial y, \partial \beta / \partial x, \partial \beta / \partial y, \partial \phi / \partial x$, and $\partial \phi / \partial y$ are calculated at the point $\left(\xi\left(\tau_{k}\right), \eta\left(\tau_{k}\right)\right), P_{+}=P\left(\xi\left(\tau_{k}^{+}\right), \eta\left(\tau_{k}^{+}\right)\right)$,
$Q_{+}=Q\left(\xi\left(\tau_{k}^{+}\right), \eta\left(\tau_{k}^{+}\right)\right) . \phi(x, y)$ is a sufficiently smooth function with grad $\phi(x, y) \neq 0$ and $\tau_{k}(k \in N)$ is the time of $k$ th jump. 
The proof of this lemma is referred to in [20].

Theorem 7. The semitrivial periodic solution $(0, \eta(t))$ of system (6) is orbitally asymptotically stable if $1-\Phi^{-1}<E_{1}$, where

$$
\begin{aligned}
\Phi & =\frac{\left(1-E_{2}\right)\left(B-1-B H\left(1-E_{2}\right)\right)}{B-1-B H} \\
& \times\left(1-B-B H\left(1-E_{2}\right)\right)^{(A(1-q)-2 B) / B}
\end{aligned}
$$

$$
\begin{aligned}
& \frac{\partial P}{\partial x}=A(1-q)(1-x-y)-1-A(1-q) x, \\
& \frac{\partial Q}{\partial x_{2}}=B(1-x-y)-1-B y, \\
& \frac{\partial \alpha}{\partial x}=-E_{1}, \\
& \frac{\partial \alpha}{\partial y}=0 \\
& \frac{\partial \beta}{\partial x}=0 \\
& \frac{\partial \beta}{\partial y}=-E_{2} \\
& \frac{\partial \phi}{\partial x}=0 \\
& \frac{\partial \phi}{\partial y}=1 .
\end{aligned}
$$

$$
\begin{aligned}
\Delta_{1} & =\frac{P_{+}((\partial \beta / \partial y)(\partial \phi / \partial x)-(\partial \beta / \partial x)(\partial \phi / \partial y)+(\partial \phi / \partial x))+Q_{+}((\partial \alpha / \partial x)(\partial \phi / \partial y)-(\partial \alpha / \partial y)(\partial \phi / \partial x)+(\partial \phi / \partial y))}{(P(\partial \phi / \partial x)+Q(\partial \phi / \partial y))} \\
& =\frac{Q_{+}\left(\xi\left(T^{+}\right), \eta\left(T^{+}\right)\right)\left(1-E_{1}\right)}{Q(\xi(T), \eta(T))}=\frac{\left(1-E_{1}\right)\left(1-E_{2}\right)\left(B-1-B H\left(1-E_{2}\right)\right)}{B-1-B H}
\end{aligned}
$$

Proof. Since $P(x, y)=x(A(1-q)(1-x-y)-1)$ and $Q(x, y)=$ $y(B(1-x-y)-1)+A q(1-x-y) x, \alpha(x, y)=-E_{1} x, \beta(x, y)=$ $-E_{2} y, \phi(x, y)=y-H$. Using Lemma 6 , we obtain and

$$
\begin{aligned}
\exp & \int_{0}^{T}\left(\frac{\partial P}{\partial x}(\xi(t), \eta(t))+\frac{\partial Q}{\partial y}(\xi(t), \eta(t))\right) d t \\
& =\exp \int_{0}^{T}(A(1-q)+B-2 \\
& -(A(1-q)-2 B) \eta(t)) d t=(1-B-B H(1 \\
& \left.\left.-E_{2}\right)\right)^{(A(1-q)-2 B) / B} \\
& \cdot\left(\frac{B-1-B H\left(1-E_{2}\right)}{(B-1-B H)\left(1-E_{2}\right)}\right)^{(A(1-q)+B-2) /(B-1)}
\end{aligned}
$$




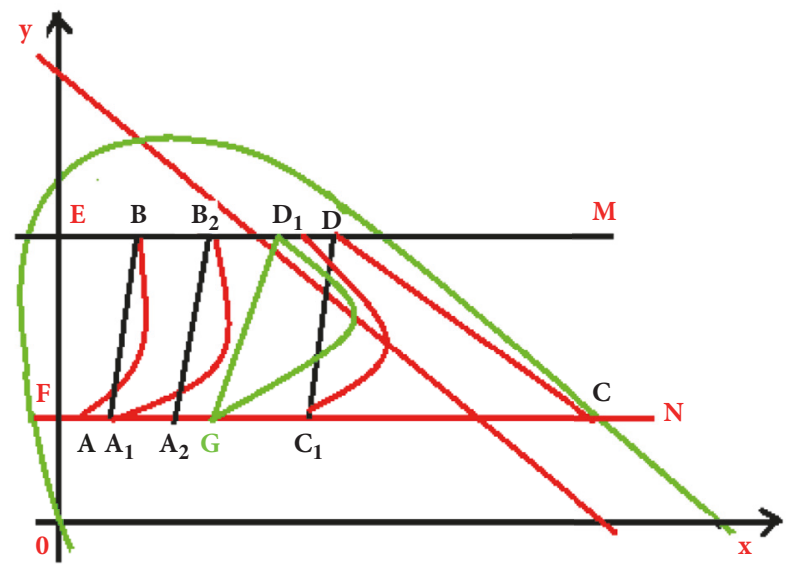

FIGURE 1: The existence of order-1 periodic solution of system (6) for $H<y^{*}$.

Therefore, if $|\mu|<1$ holds, the semitrivial periodic solution $(0, \eta(t))$ is orbitally asymptotically stable.

Remark. Defining $E_{1}^{*}=\Phi^{-1}-1$, we obtain that a fold bifurcation occurs at $E_{1}=E_{1}^{*}$ due to $|\mu|=1$, and a positive periodic solution may emerge for $E_{1}<E_{1}^{*}$.

Case 2 (existence and stability of the positive periodic solution). In this section, we denote the impulsive set $M=$ $\left\{(x, y) \in R_{+}^{2} \mid x \geq 0, y=H\right\}$, the impulsive function $\varphi(x, H)=\left(\left(1-E_{1}\right) x,\left(1-E_{2}\right) H\right)$, and the phase set $N=$ $\left\{(x, y) \in R_{+}^{2} \mid x \geq 0, y=\left(1-E_{2}\right) H\right\}$. In the following, we will prove the existence and stability of the order-1 periodic solution of system (6).

Obviously, the trajectories starting from the region $y>$ $y^{*}$ will tend to the positive equilibrium $E\left(x^{*}, y^{*}\right)$ after impulsive effect of at most finite times.

Theorem 8. If $H<y^{*}$, then system (6) has an order-1 periodic solution.

Proof. Suppose the impulsive set $M$ intersects the $y$-axis at the point $E$. The phase set $N$ intersects the $x$-axis at the point $F$ and the isoclinal line $d y / d t=0$ at the point $C$. Suppose the point $A$ is close enough to the point $F$ and the trajectory starting from the point $A$ intersects the impulsive set $M$ at the point $B$ and reaches the point $A_{1}$ due to the impulsive effect $\Delta x=-E_{1} x, \Delta y=-E_{2} y$. The point $A_{1}$ is the successor point of $A$. Thus the successor function of $A$ satisfies $f(A)=x_{A_{1}}-x_{A}>0$. Similarly, the trajectory from the point $C$ inevitably intersects the impulsive set at the point $D$. The point $D$ is mapped into the point $C_{1}$ after pulses. Furthermore, point $C_{1}$ is surely on the left of point $C$ from the property of the vector field; hence the successor function of the point $C$ becomes $f(C)=x_{C_{1}}-x_{C}<0$. According to the continuity of the successor function, we obtain that there exists a point $G$ such that $f(G)=0$; that is, system (6) presents an order-1 periodic solution (see Figure 1).
In the following, we will investigate the stability of the order-1 periodic solution by means of stability of the limit cycle of continuous dynamic system. We consider the general continuous system as follows:

$$
\begin{aligned}
& \frac{d x}{d t}=P(x, y), \\
& \frac{d y}{d t}=Q(x, y),
\end{aligned}
$$

where functions $P(x, y)$ and $Q(x, y)$ are continuous and derivative about $x, y$.

The stability of the limit cycle is investigated by Poincaré map [21] and we give some theorems as follows.

Lemma 9 (see [21]). Suppose the function $\bar{s}=f(s)$ is a continuous map from the line segment $N$ to itself and $s=0$ is a fixed point of the continuous map $f(s)$. Then the fixed point $s=0$ is stable(unstable) if the part near origin of curve $\bar{s}=f(s)$ on the plane $(s, \bar{s})$ lies in the interior of the domain $|\bar{s} / s| \leq 1-\varepsilon(\geq 1+\varepsilon), \varepsilon>0$.

Lemma 10 (see [21]). Suppose $\bar{s}=f(s)$ is continuous and derivative at the neighborhood of the point $s=0$; then the fixed point $s=0$ is stable (unstable) for $|d \bar{s} / d s|<1(>1)$.

The detailed proofs of Lemmas 9 and 10 can be seen in [21].

Since the order-1 periodic solution is not a closed curve, we will make good use of the method of the square approximation to investigate the stability of the order-1 periodic solution.

Theorem 11. Suppose $l$ is the length of the closed orbit $\bar{\Gamma}$ of system (6) and the closed order-1 periodic limit $\bar{\Gamma}$ is stable for $\int_{0}^{l} H(s) d s<1$.

Proof. Let $\Gamma_{A B C}$ denote the order-1 periodic solution of system (6) (see Figure 2(a)). The impulsive orbit $\Gamma_{A B C}$ intersects the impulsive set $M$ at point $A$ and then jumps into the phase set $N$, and intersection point is $B$. The closed orbit including the curve $\Gamma_{B C A}$ and line segment $A B$ is a closed order-1 periodic limit, which is denoted as $\bar{\Gamma}$ (see Figure 2(a)). Draw normal line $n$ passing through $B \in \Gamma$ and establish coordinate system $(s, n)$ on point $B$. Choose any point $D \in U(A, \varepsilon), \varepsilon>0$ and the trajectory starting from the point $D$ intersects vertically $n$-axis at point $B_{k}$ and then intersects impulsive set $M$ at the point $F$. The point $F$ is mapped into the point $E$ due to impulsive effect. The trajectory passing through point $E$ intersects vertically $n$-axis at $B_{k+1}$ as $t$ increases again.

Suppose the rectangular coordinate of $B$ is $(\varphi(s), \psi(s))$; we can obtain the relation of the point $B_{k}$ between the rectangular coordinates $(x, y)$ and curvilinear coordinates $(s, n)$ by using the geometric method.

$$
\begin{aligned}
& x=\varphi(s)-n \psi^{\prime}(s), \\
& y=\psi(s)+n \varphi^{\prime}(s),
\end{aligned}
$$




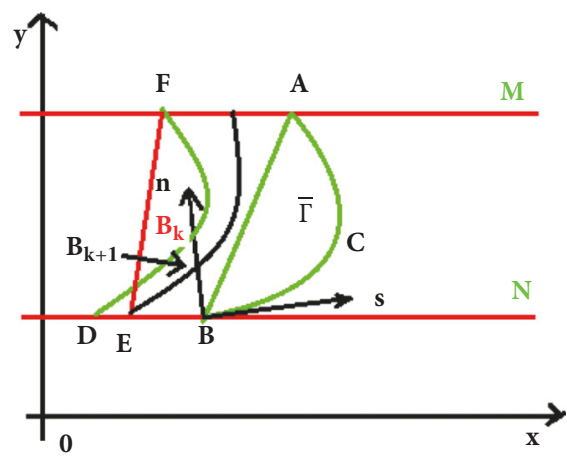

(a)

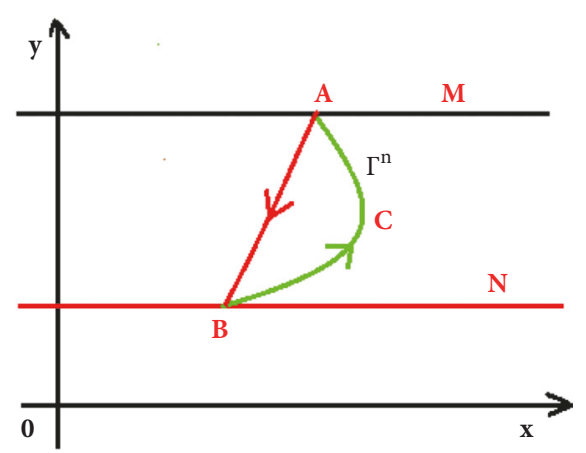

(b)

FIGURE 2: The stability of the order-1 periodic solution of system (3) for $x_{2}<x_{2}^{*}$. (b) $\Gamma^{n}$ is the order-1 periodic solution of system (32).

where $\varphi^{\prime}(s)=\left.(d x / d s)\right|_{B}=P_{0} / \sqrt{P_{0}^{2}+Q_{0}^{2}}, \psi^{\prime}(s)=$ $\left.(d y / d s)\right|_{B}=Q_{0} / \sqrt{P_{0}^{2}+Q_{0}^{2}} . P_{0}$ and $Q_{0}$ are values of $P, Q$ at the point $B$; that is, $P_{0}=P(\varphi(s), \psi(s)), Q_{0}=Q(\varphi(s), \psi(s))$.

From (28), we deduce that $d y / d x=\left(\psi^{\prime}(s)+\right.$ $\left.\varphi^{\prime}(s)(d n / d s)+n \varphi^{\prime \prime}(s)\right) /\left(\varphi^{\prime}(s)-\psi^{\prime}(s)(d n / d s)-n \psi^{\prime \prime}(s)\right)=$ $Q\left(\varphi(s)-n \psi^{\prime}(s), \psi(s)+n \varphi^{\prime}(s)\right) / P\left(\varphi(s)-n \psi^{\prime}(s), \psi(s)+n \varphi^{\prime}(s)\right)$, and we obtain

$$
\frac{d n}{d s}=\frac{Q \varphi^{\prime}-P \psi^{\prime}-n\left(P \varphi^{\prime \prime}+Q \psi^{\prime \prime}\right)}{P \varphi^{\prime}+Q \psi^{\prime}} \triangleq F(s, n) .
$$

It is easy to obtain that $n=0$ is a special solution of system (29). Since functions $P$ and $Q$ are continuous and derivative, $F(s, n)$ presents continuous first-order partial derivative about the parameter $n$. Thus, the Taylor expansion of $F(s, n)$ is given as follows:

$$
\frac{d n}{d s}=\left.F_{n}^{\prime}(s, n)\right|_{n=0} \cdot n+o(n),
$$

where $F_{n}^{\prime}(s, n)=(d / d n)(F(s, n))$. By computing, we have

$$
\begin{aligned}
\left.F_{n}^{\prime}(s, n)\right|_{n=0} & =\frac{P_{0}^{2} Q_{y 0}-P_{0} Q_{0}\left(P_{y 0}+Q_{x 0}\right)+Q_{0}^{2} P_{x 0}}{\left(P_{0}^{2}+Q_{0}^{2}\right)^{3 / 2}} \\
& \triangleq H(s),
\end{aligned}
$$

where $P_{y 0}, P_{x 0}, Q_{y 0}$, and $Q_{x 0}$ denote partial derivatives of $P, Q$ when $n=0$.

Therefore, (29) becomes $d n / d s=H(s) n$, and we obtain $n(s)=n_{0} \exp \left(\int_{0}^{l} H(s) d s\right), n(0)=n_{0}$, where $l$ is the length of the curve.

In Figure 2(a), $n_{0}$ represents the ordinate of $B_{k}$, and $n$ denotes the ordinate of $B_{k+1}$. The Poincaré map will map $B_{k}$ into $B_{k+1}$ and the successor function $f\left(n_{0}\right)=n$. According to Lemma 9 and Lemma 10, if $\left|n / n_{0}\right|<1$, the curve $B_{k} E F B_{K+1}$ starting from the neighborhood of point $B$ will tend to the closed order-1 periodic limit $\bar{\Gamma}_{A B C}$, as $k \longrightarrow \infty$. From Lemma 9 and Lemma 10, we can obtain that the closed order1 periodic limit is stable. The proof is completed.
According to the arc length formula $d s=\sqrt{P_{0}^{2}+Q_{0}^{2}} d t$ we obtain $\left.H(s)=\int_{0}^{\tau}\left(P_{x 0}+Q_{y 0}\right) d t-(1 / 2) \int_{0}^{\tau}(d / d t)\left[\ln P_{0}^{2}+Q_{0}^{2}\right)\right] d t$.

Corollary 12 (see [21]). Suppose the integral along the order-1 periodic solution $\Upsilon$ satisfies $H(s)<0$; then the order-1 periodic solution $\Upsilon$ is stable.

Suppose $Y$ is the $\tau$-periodic solution of the continuous dynamic system (27); we obtain that the value of the integral equals zero along the periodic solution of the continuous dynamic system (27); that is, $J_{\Upsilon}=\int_{0}^{\tau}(1 / 2)(d / d t)\left[\ln \left(P_{0}^{2}+\right.\right.$ $\left.\left.Q_{0}^{2}\right)\right] d t=0$. Next we will prove that the value of integral along the closed order-1 periodic limit $\bar{\Gamma}$ has similar result. Denote $F(x, y)=(1 / 2)(d / d t)\left[\ln \left(P_{0}^{2}+Q_{0}^{2}\right)\right]$.

Lemma 13. Assume the function $F(x, y)$ is continuous and differentiable; then the integral along the closed order-1 periodic limit $\Gamma$ of system (6) satisfies $\int_{0}^{\tau}(d F(x, y) / d t)=0$, where $\tau$ is the period of the closed order-1 periodic limit $\bar{\Gamma}$.

Proof. Suppose $\bar{\Gamma}$ is a closed order-1 periodic limit of system (6). $\Gamma_{\overparen{B C A}}$ denotes the curve of system (6) from the point $B\left(x_{B}, y_{B}\right)$ to the point $A\left(x_{A}, y_{A}\right)$, where $\Gamma_{\overparen{B C A}}(0)=B$, $S_{\overparen{B C A}}(T)=A$. $\Gamma_{A B}$ denotes the line segment $\overline{A B}$. Make a time transformation $\widetilde{\tau}=((n-1) / n) \tau$, and system (6) becomes

$$
\begin{aligned}
& \frac{d x}{d \tau}=x(A(1-q)(1-x-y)-1), \\
& \frac{d y}{d \tau}=y(B(1-x-y)-1)+A q(1-x-y) x, \\
& x_{2}<H,
\end{aligned}
$$

$$
\begin{aligned}
& \Delta x=-E_{1} x, \\
& \Delta y=-E_{1} y,
\end{aligned}
$$

$$
x_{2}=H .
$$

Let $\Gamma^{n}$ denote the closed order-1 periodic limit of system (32). Since system (32) is topologically equivalent to system (6), the stability of the closed order-1 periodic limit $\Gamma^{n}$ is similar to $\bar{\Gamma}$. 


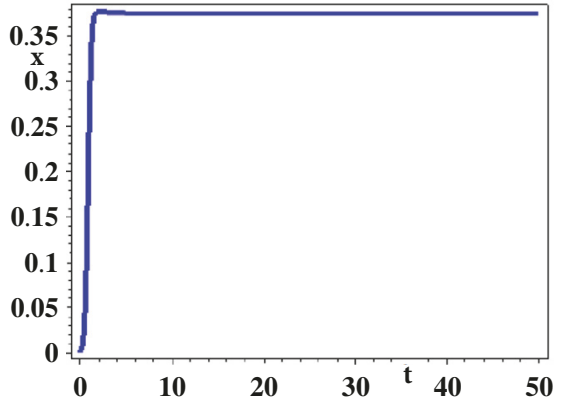

(a)

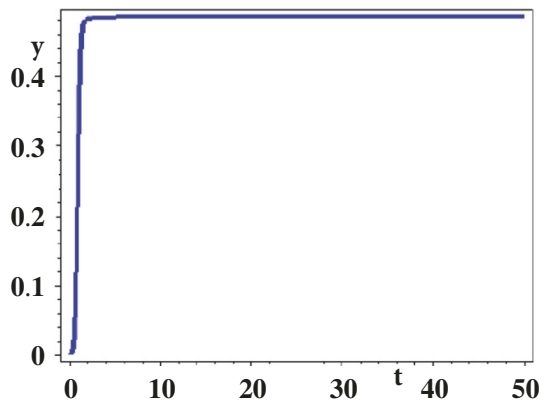

(b)

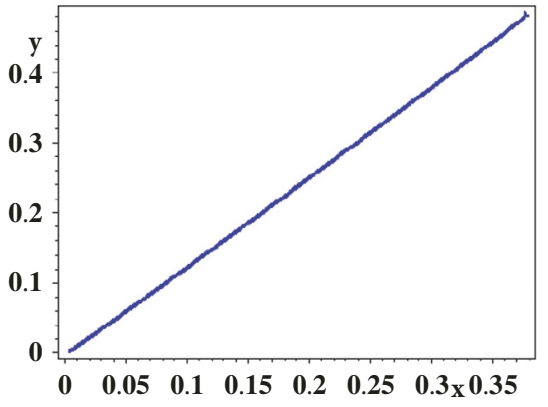

(c)

Figure 3: Time series and phase portrait of the globally asymptotical stability of the positive equilibrium $\left(x^{*}, y^{*}\right)$ with the parameters $A=$ $12, q=0.4, B=3.5$.

Suppose $\overline{\Gamma^{n}} \overline{B C A}$ denotes the curve of system (32) from the point $B\left(x_{B}, y_{B}\right)$ to the point $A\left(x_{A}, y_{A}\right)$, where $\overline{\Gamma^{n}} \widehat{\Gamma_{C A}}(0)=B$, ${\overline{\Gamma^{n}}}_{\overparen{B C A}}(((n-1) / n) \tau)=A \cdot{\overline{\Gamma^{n}}}_{A B}$ denotes the line segment $\overline{A B}$. Equation of the line segment $A B$ is $y=\left(\left(y_{A}-y_{B}\right) /\left(x_{A}-\right.\right.$ $\left.\left.x_{B}\right)\right)\left(x-x_{A}\right)+y_{A}, x_{B} \leq x \leq x_{A}$. Obviously, system (32) is the square approximation of system (6), which implies $\Gamma^{n} \longrightarrow \Gamma$ as $t \longrightarrow \infty$. Thus

$$
\begin{aligned}
\oint_{0}^{\tau} \frac{d F(x, y)}{d t} d t= & \int_{0}^{((n-1) / n) \tau} \frac{d F(x, y)}{d t} d t \\
= & \frac{1}{2} \int_{B C A} \frac{d \ln \left(P_{0}^{2}+Q_{0}^{2}\right)}{d t} d t \\
& +\frac{1}{2} \int_{A B} \frac{d \ln \left(P_{0}^{2}+Q_{0}^{2}\right)}{d t} d t \longrightarrow 0,
\end{aligned}
$$

$n \longrightarrow \infty$, which shows $\int_{0}^{\tau}(d F(x, y) / d t) d t=0$.

Therefore, we have the following result.

Theorem 14. Assume the integral along the order-1 periodic solution $\Gamma$ of system (6) has

$$
\int_{0}^{T}\left(P_{x 0}+Q_{y 0}\right) d t<0
$$

then the order-1 periodic solution $\Gamma$ is stable.

Theorem 15. The order-1 periodic solution of system (6) is stable.

Proof. From the first two equations, we have

$$
\begin{aligned}
\frac{\partial P}{\partial x}+\frac{\partial Q}{\partial y}= & A(1-q)(1-x-y)-1-A(1-q) x \\
& +B(1-x-y)-1-A q x .
\end{aligned}
$$

Sign of system (35) is uncertainty and we make an equivalent transformation $v(x, y)=1 / x y$ using the Dulac's theorem
[19]; system (6) is topologically equivalent to the following system:

$$
\begin{aligned}
\frac{d x}{d \tilde{\tau}} & =\frac{A(1-q)(1-x-y)-1}{y}=P_{1}(x, y), \\
\frac{d y}{d \tau} & =\frac{B(1-x-y)-1}{x}+\frac{A q(1-x-y)}{y} \\
& =Q_{1}(x, y) .
\end{aligned}
$$

We can compute $\partial P_{1} / \partial x+\partial Q_{1} / \partial y=-(A(1-q) / y+B / x+$ $\left.A q(1-x) / y^{2}\right)<0$. Using Theorem 14, we obtain that the order-1 periodic solution is stable.

\section{Discussion}

The ultimate goal of the genetic alteration produces good desired products by introducing a recombinant DNA into the cell in the form of a plasmid. However, the plasmid is easily lost in the reproductive process, which will bring some negative effects on the desired products. Therefore, how to effectively control the concentration of the plasmid-free organism is important to improve the efficiency of the desired products. In this paper, we formulate a mathematical model of competition between plasmid-bearing and plasmid-free organism in order to answer the question of how to reduce the negative effect of the plasmid-free organism on the plasmidbearing organism by means of the impulsive state feedback control. The positive equilibrium $E\left(x^{*}, y^{*}\right)$ of system (7) is a globally asymptotically stable, which is simulated in Figure 3 with the parameters $A=12, q=0.4, B=3.5, E\left(x^{*}, y^{*}\right)=$ $(0.3748366014,0.4862745100)$. From the practical view of production, plasmid-free organism will bring a loss of production by consuming the nutrient. Figure 3 shows that the concentration of the plasmid-bearing organism reaches the maximum value $x^{*}=0.3748366014$, while the concentration of the plasmid-free organism still increases and reaches the maximum value $y^{*}=0.4862745100$. Hence, the impulsive state feedback control must be carried out to prohibit the rapid growth of the plasmid-free organism. In Theorem 7 , the semitrivial periodic solution $(0, \eta(t))$ of system (6) is orbitally asymptotically stable if $1-\Phi^{-1}<E_{1}$ (see Figure 4 ) and the 


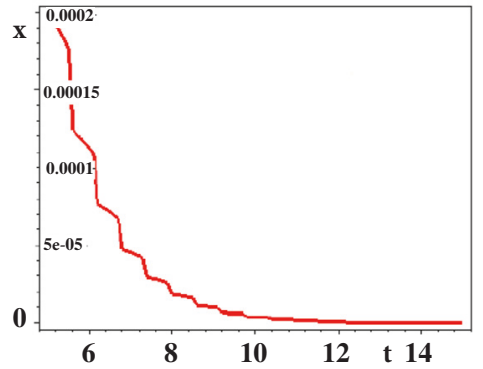

(a)

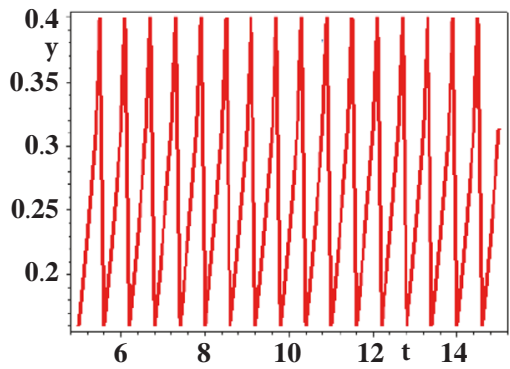

(b)

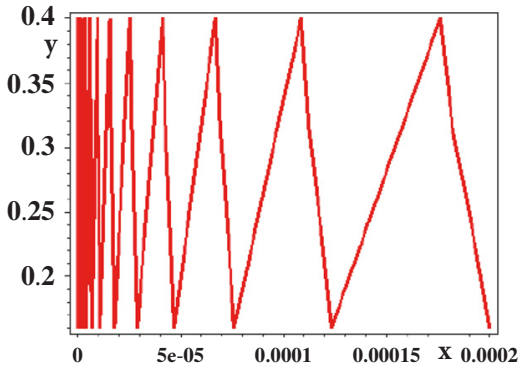

(c)

Figure 4: Time series and phase portrait of the semitrivial periodic solution of system (6) with the parameters $A=5, q=0.3, B=3.5$, $H=0.4, E_{1}=0.3, E_{2}=0.6$.

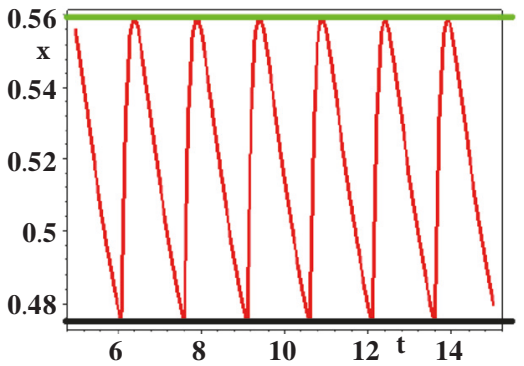

(a)

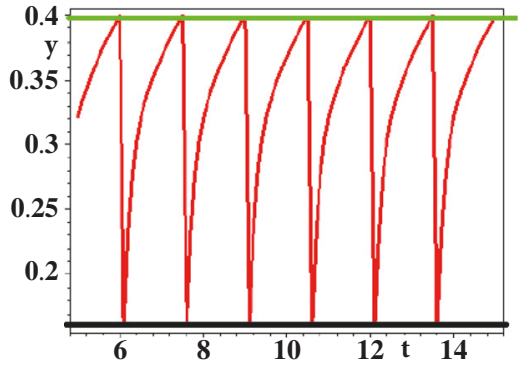

(b)

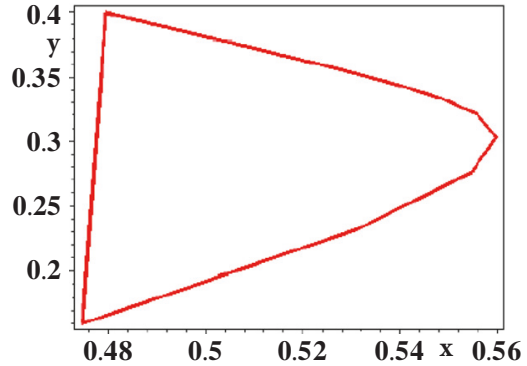

(c)

Figure 5: Time series and phase portrait of the order-1 periodic solution of system (6) with the parameters $A=12, q=0.4, B=3.5$, $H=0.3748366014, E_{1}=0.01, E_{2}=0.6$.

parameters are given $A=5, q=0.3, B=3.5, H=0.4, E_{1}=$ $0.3, E_{2}=0.6$. Theorem 7 and Figure 4 show that the plasmidbearing organism will become extinct if the extraction ratio of the plasmid-bearing organism is higher than $E^{*}=1-\Phi^{-1}$. Hence, we should try to reduce the effect on the plasmidbearing organism when we implement the impulsive state feedback control on the plasmid-free organism.

To effectively inhibit the negative effect of the plasmidfree organisms on the plasmid-bearing organism, the existence and stability of the order-1 periodic solution are given in Theorem 8 and Theorem 15. Let the parameters $A=$ $12, q=0.4, B=3.5$ be the same as Figure 3 and $E_{1}=$ $0.01, E_{3}=0.6$. In view of Figure 3 , we choose the critical threshold value $H=0.3748366014$ and simulate existence of the order-1 periodic solution in Figure 5. We show that the impulsive state feedback control is carried out once the growth rate of the plasmid-bearing organism reaches the maximum value and the negative effect of the plasmid-free organism is minimal. From Figure 5, we see that the value of the plasmid-bearing organism is $0.45 \leq x \leq 0.58$ and the value of the plasmid-bearing organism is $0.15 \leq y \leq 0.42$ under the condition of the impulsive state feedback control. Comparing Figure 3 with Figure 5, we find that the impulsive control is more effective in maximizing the concentration of plasmid-bearing organism.

\section{Data Availability}

The data used to support the findings of this study are available from the corresponding author upon request.

\section{Conflicts of Interest}

The authors declare that they have no conflicts of interest.

\section{Acknowledgments}

This work is supported by the National Natural Science Foundation of China (No. 11371164), NSFC-Talent Training Fund of Henan (U1304104), Innovative Talents of Science and Technology Plan in Henan province (15HASTIT014), Science and Technology Planning project of Henan (182102410021), and the project of the Ministry of Education CSC.

\section{References}

[1] T. K. Luo and S. B. Hsu, "Global analysis of a model of plasmid-bearing, plasmid-free competition in a chemostat with inhibitions," Journal of Mathematical Biology, vol. 34, no. 1, pp. 41-76, 1995.

[2] S.-B. Hsu and P. Waltman, "Competition between plasmidbearing and plasmid-free organisms in selective media," Chemical Engineering Science, vol. 52, no. 1, pp. 23-35, 1997.

[3] S. B. Hsu, P. Waltman, and G. S. Wolkowicz, "Global analysis of a model of plasmid-bearing, plasmid-free competition in a chemostat," Journal of Mathematical Biology, vol. 32, no. 7, pp. 731-742, 1994.

[4] S. B. Hsu and P. Waltman, "Analysis of a model of two competitors in a chemostat with an external inhibitor," SIAM Journal on Applied Mathematics, vol. 52, no. 2, pp. 528-540, 1992.

[5] S. Ai, "Periodic solutions in a model of competition between plasmid-bearing and plasmid-free organisms in a chemostat 
with an inhibitor," Journal of Mathematical Biology, vol. 42, no. 1, pp. 71-94, 2001.

[6] S. L. Yuan, D. M. Xiao, and M. A. Han, "Competition between plasmid-bearing and plasmid-free organisms in a chemostat with nutrient recycling and an inhibitor," Mathematical Biosciences, vol. 202, no. 1, pp. 1-28, 2006.

[7] K. Alhumaizi, A. Alwan, and A. Ajbar, "Competition of plasmid-bearing and plasmid-free organisms in a chemostat: a study of bifurcation phenomena," Mathematical and Computer Modelling, vol. 44, no. 3-4, pp. 342-367, 2006.

[8] B. Li, Y. Kuang, and H. L. Smith, "Competition between plasmid-bearing and plasmid-free microorganisms in a chemostat with distinct removal rates," The Canadian Applied Mathematics Quarterly, vol. 7, no. 3, pp. 251-281, 1999.

[9] N. S. Dimitrova, "Optimizing the productivity in a chemostat model of plasmid-bearing plasmid-free competition: the case of general uptake functions," Wseas Transactions on Biology and Biomedicine, vol. 1, no. 10, pp. 12-21, 2013.

[10] X. Shi, X. Song, and X. Zhou, "Analysis of a model of plasmidbearing, plasmid-free competition in a pulsed chemostat," Advances in Complex Systems. A Multidisciplinary Journal, vol. 9, no. 3, pp. 263-276, 2006.

[11] K. Sun, A. Kasperski, Y. Tian, and L. Chen, "Modelling and optimization of a continuous stirred tank reactor with feedback control and pulse feeding," Chemical Engineering and Processing: Process Intensification, vol. 50, no. 7, pp. 675-686, 2011.

[12] Y. Tian, A. Kasperski, K. Sun, and L. Chen, "Theoretical approach to modelling and analysis of the bioprocess with product inhibition and impulse effect," BioSystems, vol. 104, no. 2-3, pp. 77-86, 2011.

[13] Z. Zhao, T. Wang, and L. Chen, "Dynamic analysis of a turbidostat model with the feedback control," Communications in Nonlinear Science and Numerical Simulation, vol. 15, no. 4, pp. 1028-1035, 2010.

[14] Z. X. Li, T. Y. Wang, and L. S. Chen, "Periodic solution of a chemostat model with Beddington-DeAnglis uptake function and impulsive state feedback control," Journal of Theoretical Biology, vol. 261, pp. 23-32, 2009.

[15] D. F. Ryder and D. DiBiasio, "An operational strategy for unstable recombinant DNA cultures," Biotechnology and Bioengineering, vol. 26, no. 8, pp. 942-947, 1984.

[16] V. V. Ganusov and A. V. Brilkov, "Estimating the instability parameters of plasmid-bearing cells. I. Chemostat culture," Journal of Theoretical Biology, vol. 219, no. 2, pp. 193-205, 2002.

[17] T. Imanaka and S. Aiba, "A Perspective on the Application of Genetic Engineering: Stability of Recombinant Plasmid," Annals of the New York Academy of Sciences, vol. 369, no. 1, pp. 1-14, 1981.

[18] G. Pang, L. Chen, W. Xu, and G. Fu, "A stage structure pest management model with impulsive state feedback control," Communications in Nonlinear Science and Numerical Simulation, vol. 23, no. 1-3, pp. 78-88, 2015.

[19] A. A. Andronov, E. A. Leontovich, I. I. Gordon, and A. G. Maier, Qualitative Theory of Ordinary Differential Equations, Wiley, New York, NY, USA, 1973.

[20] P. S. Simeonov and D. D. Bainov, "Orbital stability of periodic solutions of autonomous systems with impulse effect," International Journal of Systems Science, vol. 19, no. 12, pp. 2561-2585, 1988.

[21] Y. Ye, The Theory of the Limit Cycle, Shanghai Science and Technology Press, 1984. 


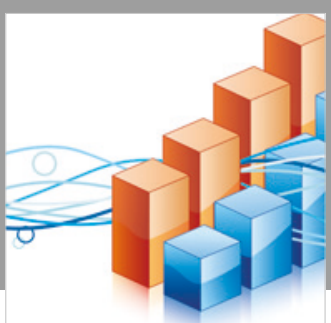

Advances in

Operations Research

\section{-n-m}
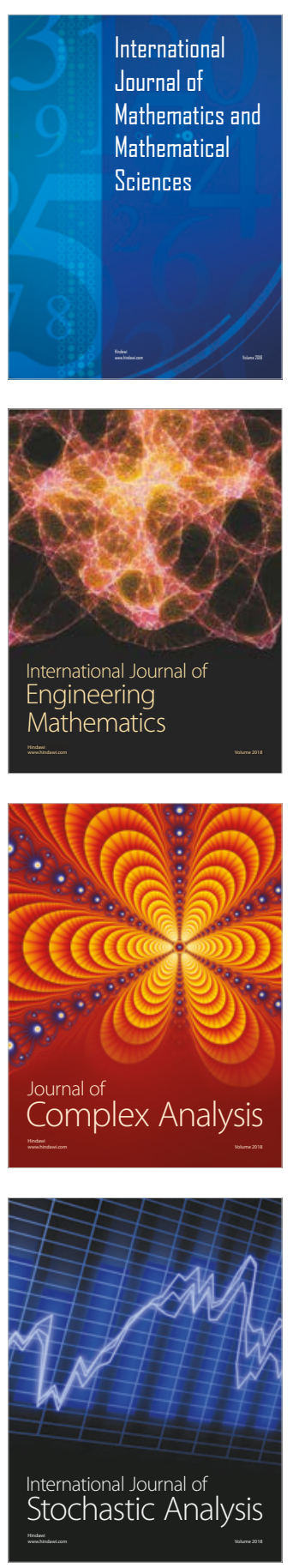
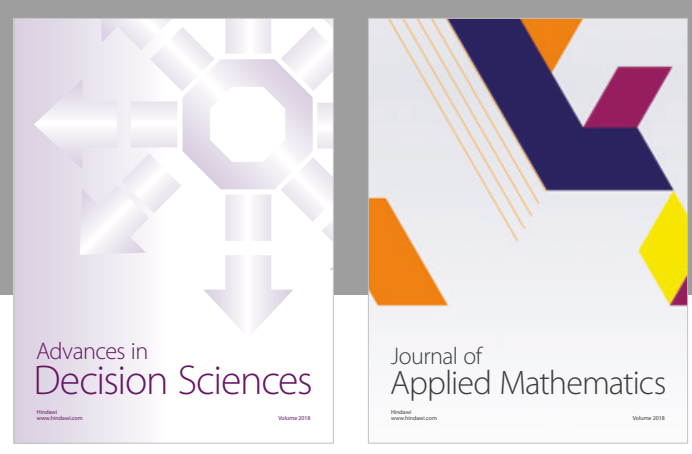

Journal of

Applied Mathematics
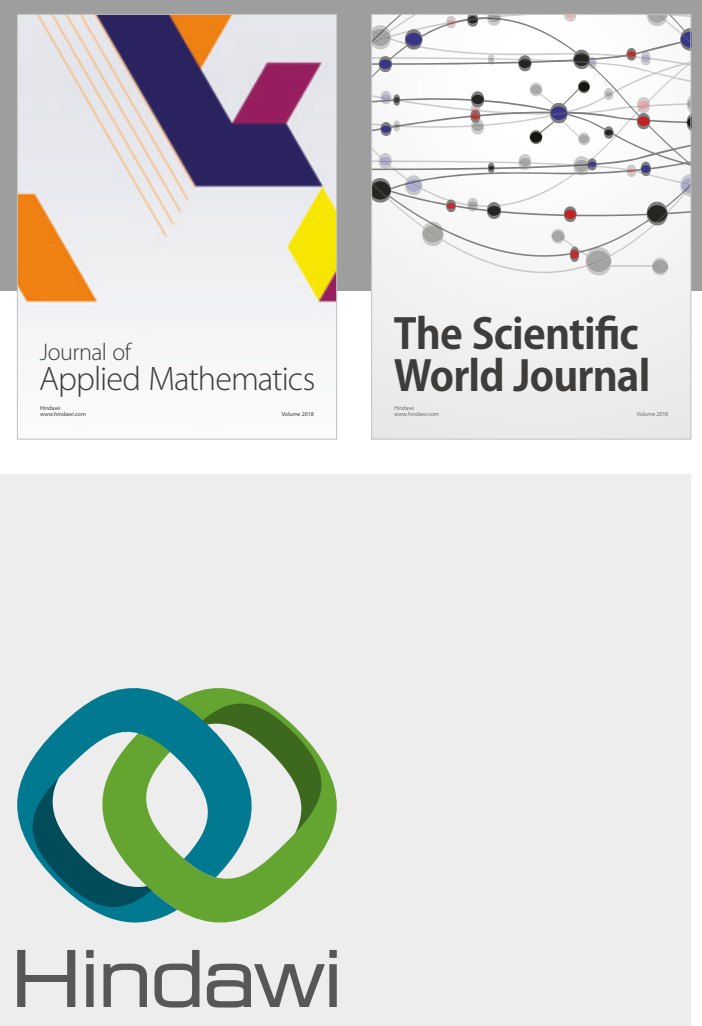

Submit your manuscripts at

www.hindawi.com

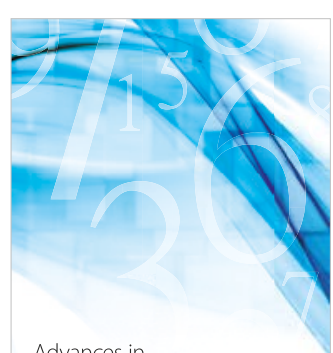

Advances in
Numerical Analysis
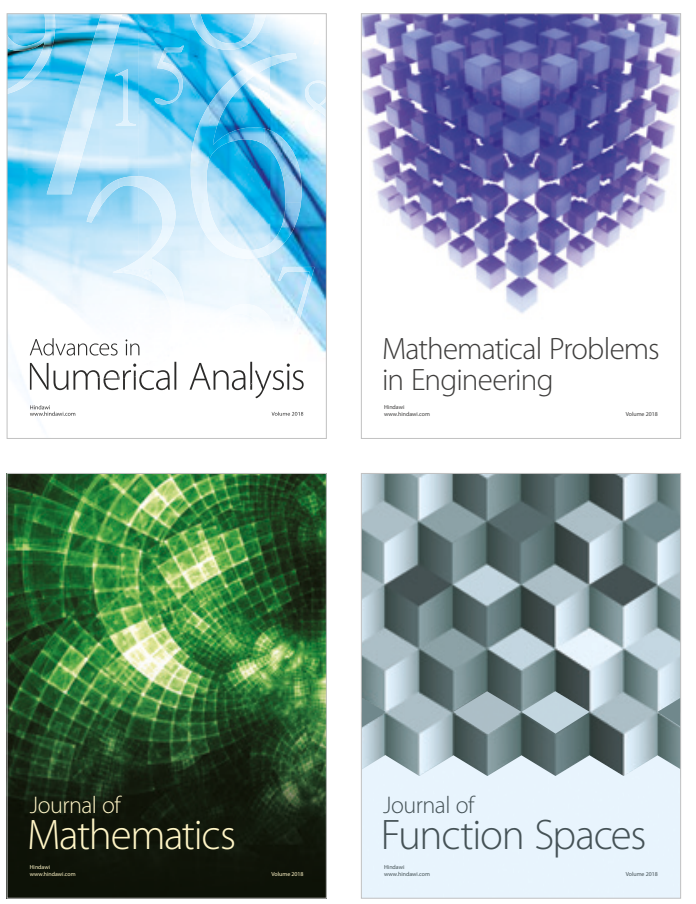

Mathematical Problems in Engineering

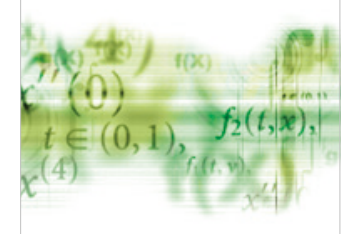

International Journal of

Differential Equations

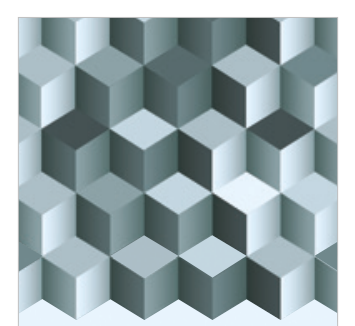

Journal of

Function Spaces

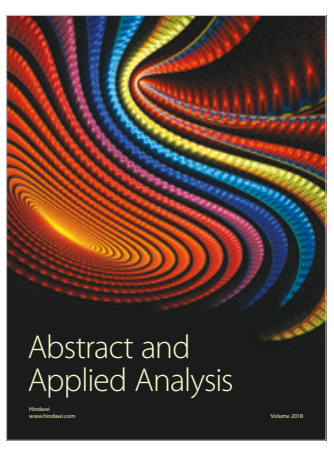

The Scientific

World Journal

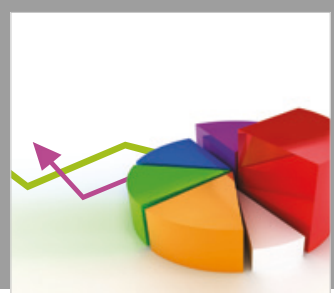

Journal of

Probability and Statistics
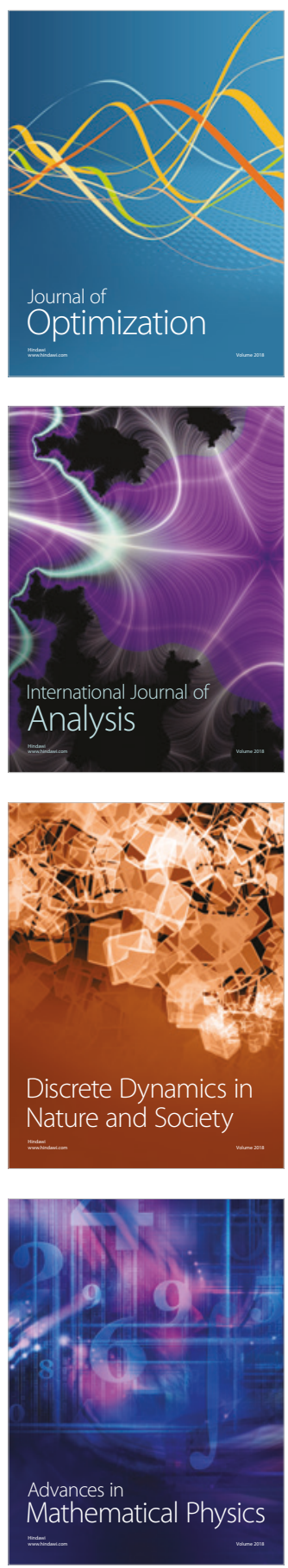